CARDIOVASCULAR MEDICINE

\title{
Relation between plasma amino-terminal propeptide of procollagen type III and left ventricular longitudinal strain in essential hypertension
}

\author{
S H Poulsen, N H Andersen, L Heickendorff, C E Mogensen
}

Heart 2005;91:624-629. doi: 10.1136/hrt.2003.029702

See end of article for authors' affiliations

Correspondence to:

Dr Steen H Poulsen

Department of Cardiology,

Skejby Hospital, Aarhus

University Hospital, DK-

8200 Aarhus N, Denmark;

steen.hviffeldt@dadlnet.dk

Accepted 15 July 2004
Objective: To investigate whether myocardial fibrosis assessed non-invasively is related to left ventricular (LV) longitudinal systolic function in patients with essential hypertension.

Design: The study consisted of 30 control subjects and 40 patients with hypertension with normal LV ejection fraction. Tissue Doppler echocardiography was performed to assess LV longitudinal systolic strain from the apical views. Mean strain was calculated from the basal and mid segments. Plasma concentrations of the amino-terminal propeptide of type III procollagen (PIIINP) were measured.

Results: In the hypertension group, mean strain was significantly reduced (mean (SD) $13(6) \%$ v 21 (6)\%, $\mathrm{p}<0.01)$ and plasma PIIINP were increased compared with controls $(3.0(0.7) \mu \mathrm{g} / \mathrm{l} \vee 2.1(0.3) \mu \mathrm{g} / \mathrm{l}$, $p<0.001)$. A significant correlation was found between mean strain and PIIINP $(r=-0.56$, $\mathrm{p}<0.001)$. In patients with abnormal diastolic filling $(\mathrm{n}=21)$ mean strain was reduced compared with patients with normal LV filling $(n=19)(10(6) \%$ v $15(6) \%, p<0.01)$ and the serological marker PIIINP was increased (3.5 (0.6) $\mu \mathrm{g} / \mathrm{l} v 2.5(0.5) \mu \mathrm{g} / \mathrm{l}, \mathrm{p}<0.001)$.

Conclusions: There is a significant association between the extent of myocardial fibrosis and reduced LV longitudinal contractility.
$\mathrm{H}$ ypertension has been found to be associated with congestive heart failure in patients with normal left ventricular (LV) ejection fraction. ${ }^{1-3}$ The presence of congestive heart failure in these patients has been attributed to isolated diastolic LV dysfunction caused by changes in myocardial relaxation and stiffness. As shown experimentally and clinically the accumulation of fibrillar collagens is exaggerated in the myocardium of the LV. This has been shown to lead to increased stiffness and abnormal cardiac function in patients with hypertension. ${ }^{4-6}$ In addition, perivascular accumulation of collagen fibres may impair the vasodilator capacity of the intramyocardial coronary arteries and contribute to impaired coronary flow reserve seen in the hypertensive heart. ${ }^{7-9}$ Non-invasive monitoring of myocardial fibrosis by use of serological markers of collagen type I and III turnover seems to provide indirect diagnostic information on the extent and the ability of antihypertensive treatment to reduce myocardial fibrosis. ${ }^{10-14}$

In most clinical and epidemiological studies of heart failure and hypertension, LV systolic performance is often considered normal by means of a normal ejection fraction or fractional shortening. However, these systolic measures do not take regional contractile function into consideration. Furthermore, outer and inner subendocardial longitudinal myocardial fibre contraction may not be clearly reflected by these traditional measures of LV systolic function. Increased fibrillar collagen deposition is believed to lead to increased interstitial and perivascular fibrosis, which is likely to affect the function of the subendocardial longitudinal oriented myocardial fibres. Tissue tracking and strain imaging are new echocardiography modalities based on Doppler tissue imaging and they may be used to assess the regional and longitudinal contractility of the LV. ${ }^{15}{ }^{16}$ Recently, we reported decreased longitudinal LV systolic function in patients with hypertension, most pronounced in patients with concomitant abnormal diastolic filling. ${ }^{17}$ We then hypothesised that in patients with arterial hypertension and normal ejection fraction, longitudinal LV contractility as assessed by tissue tracking and strain echocardiography is associated with myocardial fibrosis assessed by plasma concentrations of the amino-terminal propeptide of procollagen type III (PIIINP).

\section{METHODS \\ Participants}

The study population consisted of 40 consecutive patients with documented arterial hypertension (repeatedly increased systolic blood pressure $>140 \mathrm{~mm} \mathrm{Hg}$ and diastolic blood pressure of $>90 \mathrm{~mm} \mathrm{Hg}$ ) who were referred to our clinic for evaluation and treatment of arterial hypertension. (Blood pressure measurements shown in table 1 were obtained just before the echocardiographic examination.) Patients with secondary hypertension or a history of angina pectoris, myocardial infarction, valve heart disease, liver disease, skeletal disease, pulmonary obstructive disease, or diabetes mellitus were excluded. All patients were in sinus rhythm and with a normal LV ejection fraction (>55\%) and fractional shortening ( $\geqslant 28 \%$ ) evaluated by echocardiography. Thirty age matched normal subjects served as controls. Each study participant gave written informed consent. The study was approved by the local scientific ethics committee.

\section{Determination of plasma collagen peptide}

Blood samples to determine concentration of the markers were taken at the time of the clinical and echocardiography studies. Plasma PIIINP and carboxy-terminal telopeptides of type I collagen were analysed by radioimmunoassays (Orion Diagnostica, Oununsalo, Finland). Intracorrelation and intercorrelation variabilities for PIIINP were $<5 \%$ and $<7 \%$, respectively.

Abbreviations: $A C E$, angiotensin converting enzyme; $L V$, left ventricular; PIIINP, amino-terminal propeptide of procollagen type III 


\section{Echocardiography}

Two dimensional, $\mathrm{M}$ mode, colour $\mathrm{M}$ mode, pulsed Doppler, and tissue Doppler echocardiography was performed with a GE Vivid Five ultrasound machine (GE Medical System, Horten, Norway) with a $2.5 \mathrm{MHz}$ transducer. Echocardiograms were stored digitally and analysed with no knowledge of the clinical data. Endocardial border detection was enhanced with second harmonic imaging.

LV volumes and ejection fraction were estimated by Simpson's modified biplane method based on three measurements. $^{18}$

Pulsed Doppler measurements were obtained with the transducer in the apical four chamber view, with the Doppler beam aligned perpendicular to the plane of the mitral annulus. The sample volume was placed between the tips of the mitral leaflets. Five consecutive beats during quiet respiration were used for calculation of the Doppler variables. Colour M mode echocardiograms were recorded in the apical four chamber view with the M mode cursor aligned parallel to the LV inflow as previously described. ${ }^{18} 19$

LV mass was calculated by a validated anatomical formula and LV hypertrophy was considered present when LV mass index $>134 \mathrm{~g} / \mathrm{m}^{2}$ in men or $>110 \mathrm{~g} / \mathrm{m}^{2}$ in women. ${ }^{20}$

\section{Tissue tracking and strain imaging}

Tissue Doppler imaging (frame rate range 90-150 frames/s) was obtained from the three standard apical views during end expiration. The tissue Doppler signal angle was $<20^{\circ}$. Digitally stored loops of tissue Doppler imaging were used for offline derivation of tissue tracking images and strain recordings. Tissue tracking displays the integral of tissue velocity during systole. This equals the amplitude of motion along the Doppler axis during systole. In tissue tracking seven colour bands indicate different amplitudes of motion with a stepwise increase in motion amplitude towards the base of the $\mathrm{LV}$. In the analysis the LV apical views, the lowest motion amplitude is at the apex and the greatest motion amplitude at the mitral annulus. ${ }^{15}$ The 16 segment LV model of the American Society of Echocardiography was used to analyse the motion amplitude for each segment and the average of the 16 segments was calculated as the tissue tracking score index. ${ }^{18}$

Strain is originally defined as a dimensionless quantity produced by the application of a stress. Strain is the fractional or percentage change from the original or unstressed dimension. The tissue Doppler technique in the present study allowed processing of simultaneous tissue tracking and strain in different myocardial segments in the same cineloop. The peak systolic strain was in each segment assessed within the first $350 \mathrm{~ms}$ from the $\mathrm{R}$ wave. By definition shortening strain is expressed as negative values and stretching is reflected as positive value. The peak systolic myocardial velocity was measured in the same segments. The sample

Table 1 Clinical characteristics

\begin{tabular}{llll}
\hline & $\begin{array}{l}\text { Control } \\
\text { group }\end{array}$ & $\begin{array}{l}\text { Hypertensive } \\
\text { group }\end{array}$ & p Value \\
\hline Number & 30 & 40 & \\
Age (years) & $54(7)$ & $56(10)$ & NS \\
Smoking (yes/no) & $6 / 24$ & $12 / 28$ & NS \\
Men/women & $17 / 13$ & $21 / 19$ & NS \\
Body surface area $\left(\mathrm{m}^{2}\right)$ & $1.75(0.10)$ & $1.95(0.21)$ & 0.05 \\
Systolic BP $(\mathrm{mm} \mathrm{Hg})$ & $122(10)$ & $145(16)$ & 0.001 \\
Diastolic BP $(\mathrm{mm} \mathrm{Hg})$ & $80(9)$ & $92(11)$ & 0.001 \\
Heart rate $(\mathrm{beats} / \mathrm{min})$ & $68(9)$ & $66(10)$ & $\mathrm{NS}$ \\
Cholesterol $(\mathrm{mmol} / \mathrm{l})$ & $5.3(1.1)$ & $5.6(1.2)$ & $\mathrm{NS}$ \\
Creatinine $(\mu \mathrm{mol} / \mathrm{l})$ & $82(18)$ & $85(21)$ & $\mathrm{NS}$ \\
\hline
\end{tabular}

Data are mean (SD).

$\mathrm{BP}$, blood pressure; NS, not significant. volume was placed in the centre of the basal and mid myocardial segments in the three standard apical views and the average systolic strain was calculated for all 12 myocardial segments. The distal segments were excluded to limit the influence of the angle dependency. Tissue tracking wall score, peak systolic velocity, and strain were analysed separately and with no knowledge of two dimensional and pulsed Doppler recordings.

Interobserver variability was $\pm 6.7 \%$ for the peak systolic velocity, $\pm 5.3 \%$ for peak strain, and $\pm 4.5 \%$ for the tissue tracking score index. Intraobserver variability was $\pm 4.8 \%$ for the systolic myocardial velocity, $\pm 4.9 \%$ for strain, and $\pm 4.0 \%$ for the tissue tracking score index in 10 subjects.

\section{Diastolic LV function}

Patients were classified into two groups on the basis of the mitral inflow Doppler parameters and colour M mode flow propagation. Mitral E deceleration time $\geqslant 140 \mathrm{~ms}$ and $<240$ and an E:A ratio between 1 and 2 were considered to indicate normal diastolic filling. Abnormal diastolic filling was defined as follows: mitral deceleration time $>240 \mathrm{~ms}$ and E:A ratio $<1$ was suggestive of impaired relaxation; deceleration time $\geqslant 140 \mathrm{~ms}$ and $<240 \mathrm{~ms}$ with velocity flow propagation $<45 \mathrm{~cm} / \mathrm{s}$ was suggestive of pseudonormal filling; and deceleration time $<140 \mathrm{~ms}$ was suggestive of a restrictive filling pattern. The cut off points were chosen based on recent recommendations. ${ }^{20-22}$

\section{Statistical analysis}

All results are expressed as mean (SD). Differences between groups were tested by Student's $t$ tests for unpaired data once normality was shown; otherwise, a non-parametric test (Mann-Whitney test) was used. Categorical variables were analysed by $\chi^{2}$ test or Fisher's exact test when necessary. Correlations between variables were determined by Spearman's rank correlation test and by a multivariate regression analysis. SPSS software (version 10.0; SPSS Inc, Chicago, Illinois, USA) was used for statistical analysis and $\mathrm{p}<0.05$ was considered significant.

\section{RESULTS}

\section{Baseline characteristics}

Table 1 presents the baseline characteristics of the patients and control subjects. The median known duration of

Table 2 Echocardiographic parameters and biochemical markers of myocardial fibrosis

\begin{tabular}{|c|c|c|c|}
\hline & $\begin{array}{l}\text { Control } \\
\text { group }\end{array}$ & $\begin{array}{l}\text { Hypertensive } \\
\text { group }\end{array}$ & p Value \\
\hline IVSd (cm) & $0.9(0.1)$ & $1.2(0.3)$ & 0.001 \\
\hline LVPWd (cm) & $0.9(0.1)$ & $1.2(0.4)$ & 0.001 \\
\hline LVMI $\left(\mathrm{g} / \mathrm{m}^{2}\right)$ & $91(20)$ & $128(47)$ & 0.001 \\
\hline Ejection fraction (\%) & $62(5)$ & $62(5)$ & NS \\
\hline E:A ratio & $1.23(0.32)$ & $0.97(0.3)$ & 0.01 \\
\hline IVRT (ms) & $83(11)$ & $107(15)$ & 0.001 \\
\hline DT (ms) & $188(21)$ & $240(42)$ & 0.001 \\
\hline$V p(\mathrm{~cm} / \mathrm{s})$ & $70(18)$ & $57(18)$ & 0.001 \\
\hline $\mathrm{E} / \mathrm{Vp}$ & $1.0(0.3)$ & $1.3(0.4)$ & 0.001 \\
\hline Tissue tracking score $(\mathrm{mm})$ & $7.6(1.1)$ & $6.6(1.6)$ & 0.01 \\
\hline Mean SV $(\mathrm{cm} / \mathrm{s})$ & $5.0(1.1)$ & $4.3(1.2)$ & 0.01 \\
\hline Mean S (\%) & $21(6)$ & $13(6)$ & 0.01 \\
\hline P-PIIINP ( $\mu \mathrm{g} / \mathrm{l})$ & $2.1(0.3)$ & $3.0(0.7)$ & 0.001 \\
\hline \multicolumn{4}{|c|}{$\begin{array}{l}\text { Data are mean (SD). } \\
\text { DT, deceleration time; E/Vp, peak early velocity divided by velocity flow } \\
\text { propagation; IVRT, isovolumetric relaxation time; IVSd, interventricular } \\
\text { septal dimension; LVMI, left ventricular mass index; LVPWd, left } \\
\text { ventricular posterior wall dimension; mean S, average strain of } 12 \text { basa } \\
\text { and mid-myocardial segments; mean SV, average systolic myocardial } \\
\text { velocity of } 12 \text { basal and mid-myocardial segments; P-PIINP, plasma } \\
\text { amino-terminal propeptide of procollagen type III; Vp, velocity flow } \\
\text { propagation. }\end{array}$} \\
\hline
\end{tabular}




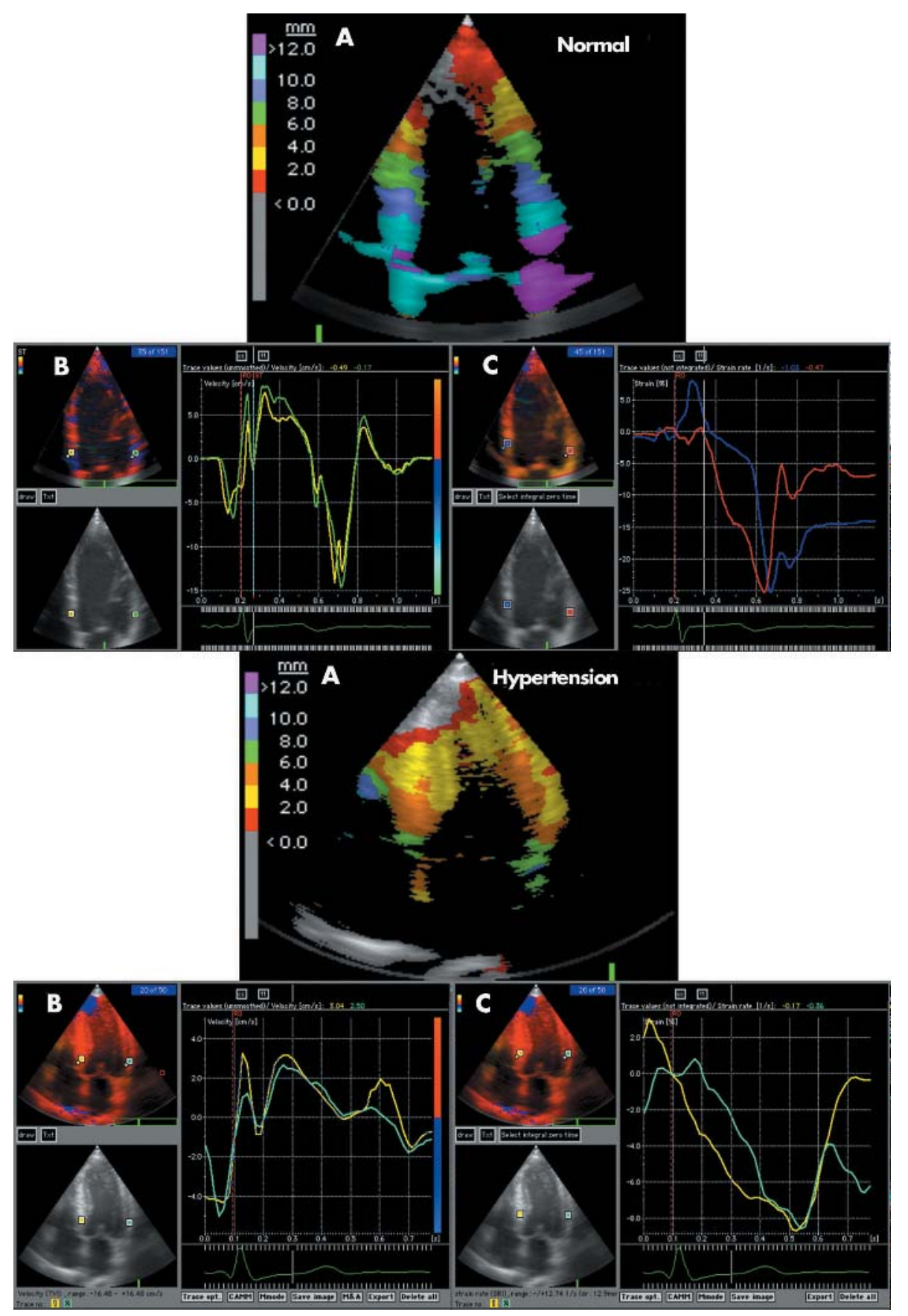

Figure 1 (A) Apical four chamber views of the left ventricle (LV) with tissue tracking analysis, which displays the longitudinal displacement (mm) from the base to the apex of the LV. (B) Peak systolic myocardial velocity and (C) strain (deformation analysis) are also shown in the septal and lateral basal segments in (top) a normal subject (plasma amino-terminal propeptide of procollagen type III (PIIINP): $2.1 \mu \mathrm{g} / \mathrm{l}$ ) and (bottom) patients with hypertension (plasma PIIINP $4.9 \mu \mathrm{g} / \mathrm{l}$ ).

hypertension was 4.2 (2.1) years (range $1-13$ years). $\beta$ Blockers were used by $40 \%$ of patients, diuretics by $50 \%$, calcium antagonist by $40 \%$, and angiotensin converting enzyme (ACE) inhibitors or ACE receptor antagonists by $38 \%$ as blood pressure lowering treatment. Thirteen patients were taking only one drug.

\section{LV function}

Table 2 shows results of the conventional echocardiography measurements and tissue Doppler analysis of LV systolic and diastolic function in the hypertension group and control subjects. LV wall thickness and mass index and indices of diastolic function were, as expected, significantly different (table 2). LV hypertrophy according to the present definition was found in 19 patients. LV systolic longitudinal function was significantly reduced in the hypertensive group compared with controls. Tissue Doppler analysis showed that the average systolic myocardial velocity and the tissue tracking score index were significantly decreased in the hypertensive group. Strain analysis showed significantly decreased deformation of the long axis function of the LV in hypertensive patients compared with controls. Figure 1 shows an example of tissue Doppler analysis of the longitudinal systolic function in a normal subject and in patients with hypertension. 


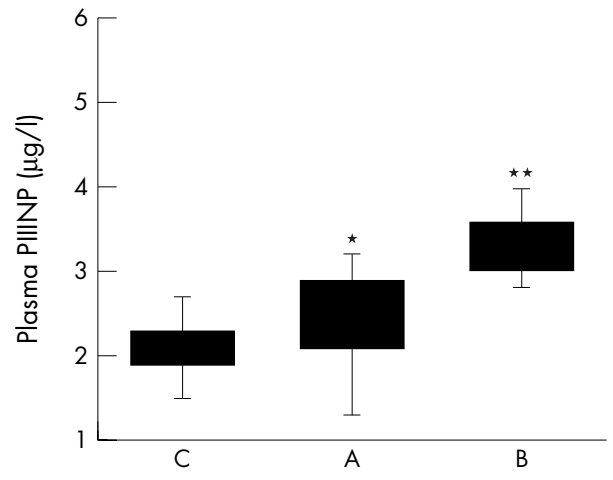

Figure 2 Comparison of plasma PIIINP between normal subjects (C) and patients with hypertension without $(A)$ or with $(B)$ abnormal diastolic left ventricular filling. ${ }^{*} p<0.01 v$ control; ${ }^{* *} p<0.01 v$ patients without abnormal LV filling.

\section{Collagen markers}

Plasma PIIINP was significantly increased in the hypertensive group as a whole compared with control subjects (table 2). Analysis of the hypertensive group showed that patients with evidence of diastolic dysfunction had significantly higher plasma concentrations of PIIINP (3.5 (0.6) $\mu \mathrm{g} / \mathrm{l}$ $v 2.5(0.5) \mu \mathrm{g} / \mathrm{l}, \mathrm{p}<0.001)$ than patients with hypertension and normal LV filling (fig 2). In addition, patients with evidence of diastolic dysfunction had significantly decreased longitudinal strain, systolic myocardial velocity, and tissue tracking score than patients with normal diastolic filling (table 3). Subanalysis showed that patients treated with either ACE inhibitor or ACE receptor antagonist $(n=15)$ had similar concentrations of PIIINP to patients treated with other blood pressure lowering agents $(\mathrm{n}=25)(2.8(0.6) \mu \mathrm{g} / \mathrm{l}$ $v 3.1(1.0) \mu \mathrm{g} / \mathrm{l}$, not significant). However, mean strain was significantly higher in patients treated with ACE inhibitor or ACE receptor antagonist than in patients without this medication (15.5 (4.0)\% $v 12.7$ (3.7)\%, p < 0.05), whereas no differences were observed between groups regarding mean systolic myocardial velocity, tissue tracking score, LV mass index, age, or blood pressure.

\section{Relation between collagen marker and LV function}

A direct significant negative correlation was found between mean longitudinal strain and PIIINP in patients with hypertension (fig 3). The tissue tracking index score correlated significantly with PIIINP $(r=-0.32, \mathrm{p}<0.05)$. Mean peak systolic myocardial velocity correlated significantly with PIIINP $(r=-0.34, \mathrm{p}<0.01)$. Among LV

Table 3 Echocardiographic and serological parameters in patients with hypertension without or with abnormal left ventricular diastolic filling

\begin{tabular}{|c|c|c|c|}
\hline & $\begin{array}{l}\text { Normal LV } \\
\text { filling }\end{array}$ & $\begin{array}{l}\text { Abnormal LV } \\
\text { filling }\end{array}$ & p Value \\
\hline Number & 19 & 21 & \\
\hline Systolic BP (mm Hg) & 144 (14) & $148(16)$ & NS \\
\hline Diastolic BP (mm Hg) & $91(10)$ & $93(13)$ & NS \\
\hline LVMI $\left(\mathrm{g} / \mathrm{m}^{2}\right)$ & $105(21)$ & $147(47)$ & 0.001 \\
\hline $\mathrm{DT}(\mathrm{ms})$ & $185(10)$ & 291 (49) & 0.001 \\
\hline IVRT (ms) & $98(10)$ & 118 (14) & 0.01 \\
\hline$E / V_{p}$ & $1.2(0.2)$ & $1.48(0.4)$ & 0.01 \\
\hline Ejection fraction (\%) & $63(6)$ & $61(4)$ & NS \\
\hline Tissue tracking score $(\mathrm{mm})$ & $7.5(1.7)$ & $6.1(1.1)$ & 0.001 \\
\hline Mean SV $(\mathrm{cm} / \mathrm{s})$ & $4.5(0.9)$ & $3.9(1.0)$ & 0.01 \\
\hline Mean S (\%) & $15(6)$ & $10(6)$ & 0.01 \\
\hline
\end{tabular}

diastolic measurements a significant correlation was shown between E:A ratio and PIIINP $(r=-0.49, \mathrm{p}<0.001)$ and between the mitral $\mathrm{E}$ deceleration time and PIIINP $(r=0.51, \mathrm{p}<0.001)$. A weak but significant correlation was found between LV mass index and PIIINP $(r=-0.41$, $\mathrm{p}<0.001)$.

A multivariate analysis of age, systolic blood pressure, body mass index, LV mass index, mean peak systolic velocity, mean strain, tissue tracking score index, E:A ratio, deceleration time, and isovolumetric relaxation time showed that strain correlated independently with PIIINP $(r=-0.54$, $\mathrm{p}<0.001$ ), whereas systolic blood pressure, age, body mass index, E:A ratio, mean systolic velocity, and tissue tracking index score did not correlate with PIIINP. Mitral E deceleration time $(\mathrm{p}<0.01)$ and isovolumetric relaxation $(\mathrm{p}<0.05)$ also correlated significantly with PIIINP in the multivariate model.

\section{DISCUSSION}

The main findings of this study are as follows. Firstly, LV longitudinal systolic function as expressed by strain and tissue tracking was decreased in patients with hypertension compared with matched controls, and the impairment of LV long axis systolic function was significantly correlated with the concentration of a serological marker of myocardial fibrosis. Secondly, the impairment of longitudinal systolic function and increased serological marker of fibrosis were most pronounced in patients with abnormal LV diastolic filling.

Emerging evidence holds promise for the use of radioimmunoassays of serological markers of fibrillar collagen type I and III in providing indirect diagnostic information about the extent of myocardial collagen turnover in hypertension. Reactive or reparative hypertensive myocardial fibrosis is the result of both increased collagen I and III synthesis by fibroblasts and unchanged or decreased extracellular collagen degradation. The increase in collagen content promotes abnormalities of myocardial performance involving diastolic filling, the coronary flow reserve, and possibly the contractile function of the myocytes. It has been proposed that cardiac fibrillar collagen may act as a link between the contractile element of adjacent cardiomyocytes, which may be changed qualitatively as well as quantitatively in the hypertensive heart. ${ }^{23}$

We found a significantly independent negative correlation between the plasma concentrations of PIIINP and LV long axis contractile function indicating that increased myocardial fibrosis affects the contractile function of inner and outer longitudinal oriented myocardial fibres. Furthermore, as shown by other investigators, we found decreased LV longitudinal segmental peak systolic velocities obtained by

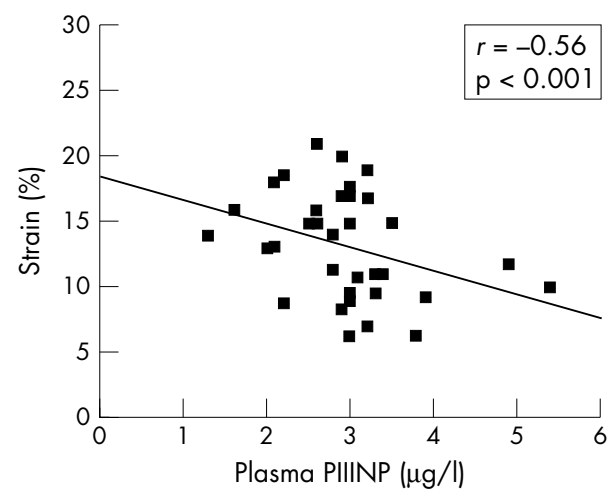

Figure 3 Correlation between average left ventricular longitudinal strain and plasma PIIINP in all hypertensive patients. 
tissue Doppler as a measure of local systolic function in patients with hypertension. ${ }^{24}$ However, this application of tissue Doppler imaging does not efficiently discriminate between actively contracting and "tethered" myocardium, where a given non-contracting segment moves in relation to the fixed transducer if it is pulled by a connected myocardial segment.

In the present study we therefore analysed LV longitudinal systolic function by strain as a measure of regional contractility, which seems to be independent of the effect of tethering and translation. Doppler echocardiography to measure myocardial longitudinal strain has had excellent correlation with tagged magnetic resonance strain measurements. ${ }^{25}$ We supplemented our evaluation of the LV longitudinal systolic function by measuring tissue tracking as an integral of regional systolic velocities describing the regional systolic amplitude motion of the LV from the base to the apex of the LV. ${ }^{15}$ This method gives the opportunity to evaluate long axis function at a glance but, as shown, the mean strain measurement was the only independent parameter associated with PIIINP, emphasising the importance of measuring longitudinal deformation by strain analysis rather than by measuring velocities.

Collagen accumulation has also been shown to affect myocardial stiffness in hypertensive heart disease. ${ }^{14}$ Accordingly, the serological collagen markers and deceleration time, a Doppler parameter known to reflect LV compliance, were independently correlated. In addition, we observed a significant reduction of LV long axis function and increased concentration of PIIINP in patients with abnormal diastolic filling compared with patients with normal diastolic filling characteristics. Abnormal mid wall fractioning of the $\mathrm{LV}$, a measure of systolic function, has been reported in patients with hypertension and normal ejection fraction. ${ }^{26}$ The presence of abnormal systolic function despite a normal ejection fraction is in accordance with our results; however, we detected abnormal longitudinal function and not radial function, which mid wall fractional shortening analysis primarily reflects. Overall, our result indicates a significant interrelation between longitudinal systolic function and diastolic function in relation to the extent of myocardial fibrosis.

In vivo and in vitro investigations have shown that the effector hormones of the renin-angiotensin-aldosterone system, angiotensin II and aldosterone, stimulate fibroblast mediated collagen synthesis. Angiotensin II may also suppress the degradation of collagen leading to progressive collagen accumulation within the cardiac interstitium. Treatment of hypertensive patients with either the ACE inhibitor lisinopril or the ACE receptor antagonist losartan has been shown to regress the amount of myocardial fibrosis measured by decreased collagen volume fraction in myocardial biopsies, to decrease plasma concentrations of the collagen marker carboxy-terminal propeptide of type I procollagen and of carboxy-terminal telopeptides of type I collagen, and to improve LV diastolic function independent of regression of LV hypertrophy. ${ }^{513-14}$ Subanalysis in the present study showed that patients treated with either an ACE inhibitor or an ACE receptor antagonist had the same concentrations of PIIINP but increased longitudinal strain compared with patients treated with diuretics, $\beta$ blockers, or calcium antagonists. Blockade of the renin-angiotensinaldosterone system seems important to reverse myocardial fibrosis and preserve myocardial function in hypertension.

Various blood pressure lowering drugs were used, which might have affected the diastolic filling parameters differently and thereby introduced the possibility of misclassifying patients regarding their diastolic functional status. However, no difference regarding the type of blood pressure lowering medication was noted between patients with and those without diastolic dysfunction. The ideal, though relatively rare, setting is clearly in patients with a new diagnosis without ongoing treatment.

\section{Study limitations}

It is clear that detectable PIIINP is not exclusively heart specific; however, the control subjects and patients with hypertension were free of liver, renal, and skeletal disease known to influence the concentrations of these markers. Although microscopic examination of endomyocardial biopsies is the most reliable method for measuring myocardial fibrosis, several studies have documented a significant correlation between histologically assessed collagen and the biochemical method based on measurements of circulating peptides derived from synthesis and degradation of fibrillar collagens.

\section{Conclusion}

LV longitudinal systolic function relates to the extent of myocardial fibrosis measured non-invasively by a serological marker of fibrosis in patients with hypertension and normal ejection fraction. The presence of isolated diastolic LV dysfunction was associated with depressed LV longitudinal systolic function and increased marker of myocardial fibrosis.

\section{Authors' affiliations}

S H Poulsen, Department of Cardiology, Skejby University Hospital, Skejby, Denmark

N H Andersen, C E Mogensen, Department of Diabetes \& Endocrinology, Aarhus University Hospital, Aarhus, Denmark

L Heickendorff, Department of Clinical Biochemistry, Aarhus

Amtssygehus University Hospital, Aarhus, Denmark

\section{REFERENCES}

1 Senni M, Tribouilloy CM, Rodeheffer RJ, et al. Congestive heart failure in the community: a study of all cases in Olmsted County, Minnesota, in 1991. Circulation 1998;98:2282-9.

2 Devereux RB, Roman MJ, Liu JE, et al. Congestive heart failure despite normal left ventricular systolic function in a population-based sample: the strong heart study. Am J Cardiol 2000;86:1090-6.

3 O'Connor CM, Gattis WA, Shaw L, et al. Clinical characteristics and long-term outcome of patients with heart failure and preserved systolic function. Am J Cardiol 2000;86:863-7.

4 Jalil JE, Doering CW, Janicki JS, et al. Fibrillar collagen and myocardial stiffness in the intact hypertrophied rat left ventricle. Circ Res 1989;64:1041-5.

5 Brilla CG, Funck RC, Rupp H. Lisinopril-mediated regression of myocardial fibrosis in patients with hypertensive heart disease. Circulation 2000;102:1388-93

6 Conrad CH, Brooks WW, Hayes JA, et al. Myocardial fibrosis and stiffness with hypertrophy and heart failure in the spontaneously hypertensive rats. Circulation 1995:91:161-70.

7 Brilla CG, Janicki JS, Weber KT. Impaired diastolic function and coronary reserve in genetic hypertension: role of interstitial fibrosis and medial thickening of intramyocardial coronary arteries. Circ Res 1991;69:107-15.

8 Kozakova M, Galetta F, Gregorini L, et al. Coronary vasodilator capacity and epicardial vessel remodelling in physiological and hypertensive hypertrophy. Hypertension 2000;36:343-9.

9 Schwartzkopff B, Brehm M, Mundhenke M, et al. Repair of coronary arterioles after treatment with perindopril in hypertensive heart disease Hypertension 2000;36:220-5.

10 Diez J, Panizo A, Gil MJ, et al. Serum markers of collagen type I metabolism in spontaneously hypertensive rats. Circulation 1996;93:1026-32.

11 Weber KT. Monitoring tissue repair and fibrosis from a distance. Circulation 1997;96:2488-92.

12 Querejeta R, Varo N, López B, et al. Serum carboxy-terminal propeptide of procollagen type $\mathrm{I}$ is a marker of myocardial fibrosis in hypertensive heart disease. Circulation 2000;101:1729-35.

13 Lopez B, Querejeta R, Varo N, et al. Usefulness of serum carboxy-terminal propeptide of procollagen type $I$ in assessment of the cardioprotective ability of antihypertensive treatment in hypertensive patients. Circulation $2001 ; 104: 286-91$

14 Diez J, Querejeta R, Lopez B, et al. Losartan-dependent regression of myocardial fibrosis is associated with reduction of left ventricular chamber stiffness in hypertensive patients. Circulation 2002;105:2512-7. 
15 Pan C, Hoffman R, Kühl H, et al. Tissue tracking allows rapid and accurate visual evaluation of left ventricular function. Eur J Echocardiogr $2001 ; 2: 197-202$

16 Urheim S, Edvardsen T, Torp H, et al. Myocardial strain by Doppler echocardiography: validation of a new method to quantify regional myocardial function. Circulation 2000;102:1158-64.

17 Poulsen SH, Andersen NH, lvarsen PI, et al. Doppler tissue imaging reveals systolic dysfunction in patients with hypertension and apparent "isolated" diastolic dysfunction. J Am Soc Echocardiogr 2003; 16:724-31.

18 Schiller NB, Shah PM, Crawford M, et al. Recommendations for quantitation of the left ventricle by two-dimensional echocardiography. J Am Soc Echocardiogr 1989;2:358-67.

19 Møller JE, Søndergaard E, Poulsen SH, et al. Pseudonormal and restrictive filling patterns predict left ventricular dilation and cardiac death after a first myocardial infarction: a serial color M-mode Doppler echocardiographic study. J Am Coll Cardiol 2000;36:1841-6.

20 Devereux RB, Lutas EM, Casale PN, et al. Standardization of M-mode echocardiographic left ventricular anatomic measurements. J Am Coll Cardiol 1984;4:1222-30.
21 Nishimura RA, Tajik AJ. Evaluation of diastolic filling of left ventricle in health and disease: Doppler echocardiography is the clinician's Rosetta stone. J Am Coll Cardiol 1997;30:8-18.

22 Garcia MJ, Thomas JD, Klein AL. New Doppler echocardiographic applications for the study of diastolic function. J Am Coll Cardiol 1998;32:865-75.

23 Gonzalez A, Lopez B, Quereta R, et al. Regulation of myocardial fibrillar collagen by angiotensin II: a role in hypertensive heart disease? J Mol Cell Cardiol 2002;34:1585-93.

24 Vinereanu D, Florescu N, Sculthorpe N, et al. Differentiation between pathologic left ventricular hypertrophy by tissue Doppler assessment of longaxis function in patients with hypertrophic cardiomyopathy or systemic hypertension and in athletes. Am J Cardiol 2001;88:53-8.

25 Edvardsen T, Gerber BL, Garot J, et al. Quantitative assessment of intrinsic regional myocardial deformation by Doppler strain rate echocardiography in humans: salidation against three-dimensional tagged magnetic resonance imaging. Circulation 2002;106:50-6.

26 Pontremoli R, Ravera M, Bezante GP, et al. Left ventricular geometry and function in patients with essential hypertension and microalbuminuria. J Hypertens 1999; 17:993-1000.

\section{IMAGES IN CARDIOLOGY}

\section{Scintigraphic diagnosis of acute pulmonary oedema}
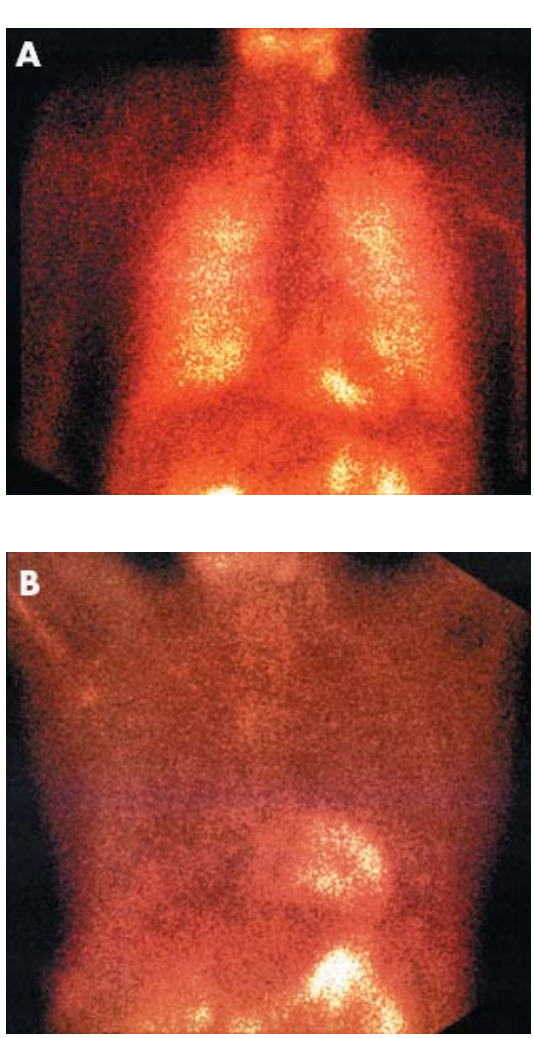

$\Lambda$ 50 year old man underwent a myocardial perfusion scan at rest for chest pain evaluation. He received an intravenous injection of Tc-99 sestamibi, while complaining of recurring angina, and underwent a single photon emission computed tomography (SPECT) scan approximately 90 minutes later. An initial planar image acquisition showed a very prominent retention of the tracer in the lungs, with a lung:heart ratio of 0.98 (normal reference $<0.44$ ) (panel A). The tomographic images of the myocardium showed severe anterior, septal and lateral hypoperfusion with significant systolic dysfunction. After image acquisition, the patient presented with worsening dyspnoea and haemoptysis compatible with evolving acute pulmonary oedema. After adequate response to medical treatment, he was sent to the cath lab, having shown a severe lesion at the left coronary ostium. He underwent coronary bypass graft surgery with subsequent complete remission of the symptoms. A control myocardial perfusion scan at rest performed eight days after surgery showed the lung:heart ratio (0.40) had returned to normal with normal segmental myocardial perfusion (panel B). This unusual presentation depicted in panel A represents severe left ventricular failure reflecting subtotal left main coronary artery occlusion, and was decisive for ensuring adequate patient management.

J N Barbisan E Ludwig pesquisa@cardiologia.org.br 